\title{
Diversificación de las estructuras familiares: caracterización de las convivencias en Chile $^{1}$
}

\author{
Catalina Arteaga A.* \\ Denisse Sepúlveda** \\ Verónica Aranda ${ }^{* * *}$
}

\begin{abstract}
Resumen
Enmarcado en el debate acerca de las distintas modalidades de familias en las sociedades contemporáneas, el presente artículo busca caracterizar las convivencias a partir de un análisis de los datos de la encuesta Casen 2006, que es la fuente más reciente y completa que permite indagar en dicho aspecto a nivel nacional. En términos generales, es posible establecer un aumento de las convivencias en desmedro de las uniones formales en los últimos años y una ampliación de dicha modalidad de unión en otros grupos sociales a los tradicionalmente observados -sectores más empobrecidos-. Asimismo, en el trabajo exploramos en algunas variables (edad, años de escolaridad, quintil, zona urbana o rural y condición de actividad) que inciden en la probabilidad de establecer la convivencia, de manera diferenciada por sexo.
\end{abstract}

Palabras clave: Familia - modernidad - convivencias - modelo de regresión logística.

\begin{abstract}
As part of the debate about different types of families in modern societies, this article aims to describe relationships through the analysis of survey data from Casen 2006, which is the latest and most comprehensive source that can inquire into this aspect at the national level. In general, in recent years it is possible to establish an increase of "premarital relations" at the expense of formal unions and an extension of such form of union in other social groups to the traditionally observed-impoverished sectors-. Furthermore, the research explores how socio-demographic variables (age, years of schooling, quintile, urban or rural area and activity status) affect the probability of establishing premarital relationships on a sex-differentiated way.
\end{abstract}

Keywords: Family - modernity - premarital relations - logistic regression model.

1 Agradecemos a Adrián Leguina por el apoyo prestado en el análisis estadístico de la información.

* Doctora en Ciencias Políticas y Sociales. Académica del Departamento de Sociología, Universidad de Chile.

** Magíster en Género y Cultura, mención Ciencias Sociales.

*** Socióloga, Universidad de Chile. Magíster Pontificia Universidad Católica de Chile. Estudios Doctorales en Ciencias Sociales, Universidad de Chile. 


\section{INTRODUCCIÓN}

Uno de los procesos propios de las sociedades modernas se relaciona con las transformaciones de las relaciones familiares. A partir de eventos como la Revolución Industrial, las ciencias sociales han atendido a la dimensión doméstica como uno de los referentes importantes de transformación social, a la vez que un espacio privilegiado de observación de cambios acontecidos en la esfera de la vida privada y la familia. En las últimas décadas, en el ámbito de estas disciplinas ha habido un renovado interés por el estudio de las familias, incidido por los nuevos enfoques metodológicos y teóricos, así como por las transformaciones sociales y el desarrollo de movimientos que han enfatizado en la importancia del ámbito privado como objeto de estudio.

El análisis de la familia constituye una de las claves más importantes para la comprensión de las relaciones entre lo público y lo privado, lo institucional y lo personal. Y es que las transformaciones modernas de la familia han tenido lugar en todo el mundo a lo largo de las principales rutas históricas que conducen hacia la modernidad y la atraviesan (Therborn, 1999). En efecto, el estudio de las familias, así como de los grupos domésticos², permite el análisis de un espacio institucional que da cuenta de dimensiones relevantes en la conformación de las relaciones sociales a un nivel macrosocial -si se atiende a las dinámicas en la conformación y cambios de estructura, composición y roles-, a la vez que permite indagar en los vínculos intersubjetivos que se dan en el interior y que están ligados a procesos de orden más estructural. El estudio de las relaciones entre el Estado y la vida privada, la vinculación entre fuerza de trabajo y mercado, el análisis de la división sexual del trabajo, las estrategias desplegadas por las familias ante efectos de crisis socioeconómicas -entre otros aspectos-, dan cuenta de procesos sociales relevantes, no siempre indagados, que permiten esclarecer las formas de relación entre dinámicas estructurales y la vida cotidiana de los individuos.

La sociología desde sus inicios como disciplina ha analizado a la familia, indagando en el papel central que ha cumplido en la sociedad desde sus múltiples funciones: como sistema de socialización y reproducción de pautas socioculturales, como ámbito de organización del trabajo y, más recientemente, como un espacio que-junto a otras instituciones socialesdota al individuo de referentes centrales para la reproducción de las identidades de sus miembros. Además del carácter integrador de este núcleo, desde una perspectiva crítica -particularmente las teorías de género- se ha analizado a la familia como un campo donde se despliegan relaciones de poder (Hartmann, 2000), reconociendo en su interior relaciones de disputa, negociación y conflicto entre sus miembros (Jelin, 1984; González de la Rocha et al., 1990; Kabeer, 1998).

En la actualidad, distintos autores se refieren a las transformaciones en el ámbito de las relaciones familiares, enmarcadas en el cambio societal contemporáneo. Así, autores como Beck (2003) se refieren a la preeminencia de la individualización, que afectaría también a

2 En términos generales, al hablar de familia en la literatura se hace referencia a grupos vinculados por el parentesco, mientras que la noción de grupo doméstico refiere a grupos unidos o no por lazos de parentesco, que corresiden. 
los miembros de la familia, señalando que se estaría transitando desde una comunidad de necesidad a un tipo de relaciones electivas, observando que la familia estaría adquiriendo una nueva forma histórica que Ilama -citando a Rosenmayr-familia posfamiliar. Por su parte, Castells (2004) hipotetiza que hoy en día es posible referirse a lo que denomina una "crisis de la familia patriarcal". Con ello se hace referencia al debilitamiento de un modelo basado en el ejercicio estable de la autoridad/dominación sobre toda la familia del hombre adulto que es su cabeza (Castells, 1999). En este contexto, el autor destaca como indicadores de esta crisis la creciente frecuencia de las crisis matrimoniales y la disolución de los hogares de las parejas casadas, por divorcio o separación; el retraso en la formación de parejas y la vida en común sin matrimonio; la variedad también creciente de estructuras de hogares, con lo que se disuelve el predominio del modelo clásico de la familia nuclear tradicional (parejas casadas en primeras nupcias y sus hijos); y la tendencia en aumento a que nazcan más niños fuera del matrimonio y a que las mujeres limiten el número de hijos y retrasen el primero (Castells, 1999). Esta nueva caracterización no implicaría la disolución de la familia como tal, sino más bien el fin de la familia como la hemos conocido hasta ahora. No sólo de la familia nuclear (un artefacto moderno), sino de la basada en la dominación patriarcal ${ }^{3}$.

En el contexto latinoamericano, desde hace varios años distintos/as autores/as han enfatizado en los cambios acontecidos en este ámbito, realizando investigaciones y estudios relevantes al respecto (De Oliveira et al., 1989; Valdés y Valdés, 2005) y dando a conocer la multiplicidad de transformaciones en la estructura y composición de las unidades domésticas, así como las tensiones, conflictos y distintos sentidos que se dan en el ámbito familiar. Como bien sintetizan Araujo y Martuccelli (2012), la discusión sobre la familia en Chile es relevante y es posible establecer dos grandes posturas: aquellas que reconocen cambios, pero relevan el carácter institucional de la familia y su capacidad de producción de orden social; y quienes, por otra parte, subrayan la desinstitucionalización de esta instancia, asociados a procesos de democratización.

Ambas posturas, no obstante, reconocen que ha habido transformaciones en esta institución. Respecto de los cambios, es posible advertir que junto a las formas tradicionales de familia han surgido nuevas configuraciones familiares como parejas sin hijos y hogares sin núcleo conyugal, a la vez que continúan aumentando los hogares monoparentales ${ }^{4}$,

3 Particularmente en América Latina, la década de los 90 fue una etapa de legitimación de la búsqueda de formas y relaciones menos patriarcales, menos autoritarias, más sensibles al reconocimiento de los derechos individuales de los miembros de las familias. Desempeñaron un rol crucial en este proceso, del cual fue parte también la Conferencia de Beijing, el movimiento de mujeres y sectores de la sociedad civil que le dieron sustento político a estos eventos internacionales. En ellos se consolidó una nueva agenda que es expresión de los consensos alcanzados en torno a nuevos derechos y a nuevos sujetos de derecho.

4 A partir de la información de encuestas de hogares, Arriagada (2004) distingue entre hogares unipersonales (una sola persona) y hogares sin núcleo (aquellos donde no existe un núcleo conyugal -una relación padre/madre e hijo/hija-, aunque puede haber otros lazos familiares). Entre los tipos de familias se distinguen: familias nucleares (padre o madre o ambos, con o sin hijos); familias extendidas (padre o madre o ambos, con o sin hijos y otros parientes) y familias compuestas (padre o madre o ambos, con o sin hijos, con o sin otros parientes y otros no parientes -excluyendo el servicio doméstico puertas adentro y sus familiares-). A su vez, las familias pueden ser biparentales (pareja, con o sin hijos) o monoparentales (con sólo un padre -habitualmente la madre- e hijos). 
especialmente los de jefatura femenina. El tamaño medio de la familia se ha reducido por la declinación del número de hijos y el mayor espaciamiento entre ellos, pero es importante indicar que esta reducción también obedece a otros fenómenos como la disminución de las familias multigeneracionales y el aumento de hogares unipersonales. El modelo de familia extendida cada vez existe menos, al tiempo que la formación de familias nucleares ha pasado a constituirse en el grupo dominante.

En este contexto, algunas de las transformaciones más relevantes en las familias en América Latina y Chile que han sido documentadas dicen relación con una disminución de la fecundidad y una reducción del tamaño promedio de la familia; el aumento de hogares con dos proveedores; los hogares con ausencia temporal o permanente de miembros por migraciones; cambios en roles de género; la diversidad de formas familiares; el aumento de separaciones y divorcios y la presencia de segundas uniones; el aumento de relaciones sexuales de los jóvenes previamente al matrimonio; y el cambio en sentido y funciones de la familia -entre otros aspectos- (Arriagada, 2001, 2004; Wainerman, 2003).

Al mismo tiempo, la familia como institución social básica no está ajena a valores culturales y a procesos políticos de cada momento o período histórico (Jelin, 2004). Las organizaciones familiares están ligadas al mercado de trabajo y a la organización de redes sociales, por lo que tendencias tales como las tasas de fecundidad y de divorcio, o los procesos de envejecimiento, son parte de procesos sociales, económicos y culturales más amplios, que están también sujetos a políticas públicas.

Cabe advertir además que la modernización se caracterizó por una creciente diferenciación y especialización de las instituciones. En el caso de la familia, ello se ha traducido en una progresiva concentración en funciones afectivas y la absorción por otras instituciones de las tradicionales funciones instrumentales. Como resultado de este proceso se debilitan los lazos de dependencia mutua entre los integrantes del núcleo familiar y las relaciones interpersonales sufren también transformaciones.

En dicho marco, diversos autores han enfatizado en los cambios sociodemográficos de la sociedad chilena en las últimas décadas, recalcando, no obstante, que muchas de estas transformaciones vienen de tiempos más remotos que los que comúnmente se argumenta. Al respecto, Valdés (2004) señala que la preeminencia de un modelo de familia moderno-industrial sancionado por el matrimonio estuvo vinculado a un Estado social protector que llevó a una disminución de los hijos nacidos fuera del matrimonio y de las uniones consensuales entre 1940 y 1970. Los procesos de institucionalización de la familia en el matrimonio derivaron de las políticas sociales y laborales del Frente Popular (Rosemblatt, 2000). No obstante, en la actualidad estaríamos asistiendo más bien a un proceso de desinstitucionalización de la familia, junto a la diversificación de patrones familiares (Valdés, 2004).

No obstante estas características, el rasgo dominante de los modos de organización de la vida familiar en la región es la diversidad. Esa diversidad incluye la coexistencia de familias extensas multigeneracionales, compuestas, nucleares con uno a ambos jefes, hogares con jefatura femenina, hogares unipersonales. Ha crecido el número de hogares en que ambos 
cónyuges trabajan y se ha producido un incremento en las relaciones prematrimoniales. Asimismo, ha aumentado el número de uniones consensuales, así como los casos de dos o más uniones sucesivas que se traducen en distintos arreglos legales y económicos para la crianza de los hijos.

En efecto, uno de los rasgos distintivos en la conformación familiar latinoamericana, y chilena en particular, dice relación con el aumento de las uniones informales, uniones consensuales o convivencias (García y Rojas, 2002; Rodríguez, 2004). Las recientes perspectivas teóricas respecto del sentido de las uniones informales, su significación y su potencial afinidad con la modernidad a largo plazo han llevado a reconsiderar esta visión de una creciente institucionalización de las uniones.

En este contexto de cambios nos preguntamos: ¿cuáles son las características de este tipo de uniones? ¿Cómo han cambiado a lo largo del tiempo en el país? ¿Qué rasgos presentan los sujetos involucrados en estas relaciones?

A partir de la revisión de estudios previos (Rodríguez Vignoli, 2004; García y Rojas, 2002), así como de la elaboración propia de datos a partir de la encuesta Casen 2006 5 , abordaremos estas interrogantes con el objeto de caracterizar el perfil de las convivencias en la actualidad y proponer algunas hipótesis vinculadas al sentido de dicho comportamiento en el contexto de los cambios sociales contemporáneos, entendiendo que la comprensión del sentido de las mismas para sus miembros, así como su relevancia en la dinámica social, implicarían llevar a cabo un estudio de más largo plazo, con mayor profundidad y la aplicación de otras técnicas de indagación.

\section{PERFIL DE LAS CONVIVENCIAS EN CHILE}

Como señalamos, en las últimas décadas las uniones heterosexuales han aumentado en toda la región. En el caso de Chile se ha producido una ampliación de éstas desde los años 80. Como se advierte en el Cuadro 2 (en Anexo), los núcleos familiares legalmente sancionados (casados) han ido en disminución, a diferencia de las convivencias que han aumentado. En el año 2006 éstas representaron el 14,9\% del total de uniones de personas mayores de 18 años en el país ${ }^{6}$.

Un elemento que sigue destacándose en el país con respecto a las características de las convivencias es que, entre los más jóvenes (18 a 25 años), ésta es mucho más recurrente que el matrimonio legal (Cerruti y Binstock, 2009). Sin embargo, advertimos que en los años recientes se ha producido un cambio en el grupo de edad que sigue, en la medida

5 La encuesta Casen del año 2006 es representativa de hogares particulares a nivel nacional, regional y por zona (urbana y rural) para 335 comunas del país. El marco muestral retomó la información de viviendas y población del Censo del año 2002. En el 2006 la muestra consideró 74.300 viviendas. Se encuestaron 73.720 hogares y un total de 268.873 personas, con un nivel de confianza del $95 \%$.

6 La encuesta Casen registra las siguientes categorías de estado civil: casado/a, conviviente o pareja, anulado/a, soltero/a, divorciado/a, separado/a y viudo/a. 
que también se perfila una importante proporción de parejas en esta situación (Cuadro 3, en Anexo). En efecto, aquellas personas que tienen pareja entre los 26 a 35 años también prefieren la convivencia como forma de unión frente al matrimonio, lo que nos habla de una mayor transversalidad de esta modalidad de pareja a otros grupos etarios. Para el adulto-joven, la convivencia aparece como una forma aceptada de constitución de familia. A partir de los grupos de mayor edad -de 36 años o más- prima la convivencia en relaciones matrimoniales, aunque ésta es más marcada desde los 46 años en adelante. El hecho de que casi un tercio de las parejas de 26 a 35 años se reúnan en relaciones de convivencia nos habla de un patrón claro de modalidad de convivencia que se ha instalado en dicho grupo.

Si analizamos las diferencias por sexo, los datos demuestran que la diferencia más notable se advierte en el grupo de 18-25 años, donde claramente las mujeres declaran convivir a edades más tempranas que los hombres, pudiendo inferir que es la mujer quien propicia la opción de otros tipos de familia antes del matrimonio legal. Ello evidencia una actitud más moderna en la constitución de tipos de familias, que se distancia de las constituciones formales más tradicionales.

Al considerar el nivel educacional, podemos apreciar que los casados presentan mayores niveles de educación superior completa $(14,4 \%)$ que los convivientes $(9,1 \%)$. Esto es posible explicarlo por la diferencia en edad entre ambos grupos; sin embargo, el grupo de convivientes presenta mayores niveles de educación media completa (22,5\% contra 20,5\% en humanística-científica y $8,5 \%$ contra $7,2 \%$ en técnica) y también mayores niveles en educación superior incompleta $(6,1 \%$ contra $4,1 \%)$. Ello nos indica que en el futuro podemos esperar que este grupo de convivientes posea mayores niveles educacionales que el grupo actual de casados, generando una mayor movilidad social para este grupo considerando la tendencia a la disminución en la cantidad de matrimonios. De esta forma, el grupo de convivientes tiene mayor potencial de ascenso en capital social y económico que el grupo de casados, mayores y establecidos.

Respecto de los sentidos de las convivencias, como señala Rodríguez (2004) los datos disponibles no permiten clarificar si la convivencia es una apuesta estratégica (alternativa al matrimonio) o táctica (prueba prematrimonial) o una situación impuesta a partir de eventos particulares (crisis económica, presencia de hijos, entre otros). Si bien la respuesta a este tipo de interrogantes pasaría por desarrollar estudios con otro tipo de enfoques, es posible hipotetizar que este tipo de uniones se está expandiendo y transversalizando como patrón de comportamiento de parejas en distintos grupos de edad y condición socioeconómica, lo que seguramente hace referencia a distintos sentidos que coexisten en una decisión de este tipo en las parejas que optan por una unión con este carácter. No obstante, es imprescindible realizar estudios de otro tipo que permitan ahondar en dichos aspectos.

\section{CONVIVENCIAS Y GRUPO SOCIAL}

Una de las cuestiones que se ha destacado en los análisis respecto a las convivencias en América Latina tiene que ver con la adscripción de clase de este tipo de pareja. En general, se ha observado que la convivencia se da más tempranamente entre los jóvenes de 
grupos más pobres (Rodríguez, 2004). Sin embargo, a partir del análisis de la Casen 2006 podemos observar que la agrupación de estados conyugales respecto a los ingresos a través del quintil autónomo nacional confirma que el grupo de casados presenta porcentajes más homogéneos entre los cinco quintiles (entre el 18\% y 20\%) que el grupo de convivientes. Éstos se concentran en los quintiles I y II (22,9\% en ambos) y tienen un menor porcentaje en el quintil V (14,6\%), evidenciando que corresponden a un grupo económicamente más débil. Sin embargo, es importante destacar que hasta el quintil IV no existen grandes diferencias al interior del grupo de convivientes; asimismo, de acuerdo con lo analizado anteriormente, es posible esperar que la diversidad sea atenuada una vez que los(as) convivientes alcancen los niveles educacionales que aún tienen incompletos, produciendo igualdad entre ambos grupos -siempre que mantengan su estado civil- (Cuadro 4, en Anexo).

Así, es posible destacar que, si bien las parejas convivientes se ubican más claramente en los primeros quintiles, hay una distribución bastante homogénea en el porcentaje de parejas de hecho entre el primer y cuarto quintil. Al respecto, algunos estudios de tipo cualitativo han señalado las diferencias de clase en las decisiones y conformaciones familiares. Al respecto, Valdés et al. (2005) -de acuerdo al análisis de 50 entrevistas de parejas y separados de ambos sexos provenientes de grupos altos, medios y bajos, de edades entre los 25 y 45 años con hijos/as- señalan que existen diferencias de unión entre las clases sociales. Encontraron que en los grupos altos y medios se tiende a institucionalizar la familia en el matrimonio civil con régimen de separación de bienes, el cual es generalmente acompañado del matrimonio religioso, mientras que la clase media presenta una mayor diversidad en la composición y coexistencia de formas de unión desde convivencias a matrimonio, pasando por los diversos regímenes matrimoniales existentes.

\section{PARTICULARIDADES DE GÉNERO EN LAS CONVIVENCIAS}

Con el objeto de ahondar en la caracterización de las convivencias de manera diferenciada por género, se desarrolló un modelo de regresión logística que buscó indagar en algunas variables específicas (edad, años de escolaridad, quintil, zona urbana o rural y condición de actividad) y su incidencia como probables elementos explicativos de la situación de convivencia ${ }^{7}$.

Específicamente en esta sección veremos el impacto de algunos factores estructurales (edad, años de escolaridad, quintil, zona urbana o rural y condición de actividad) sobre la decisión que tiene una persona de ser conviviente o casado. Dentro de la variable estado conyugal se consideraron sólo las categorías convivientes y casados porque el resto de las categorías (solteros, viudos, separados, divorciados) no corresponde a una relación de pareja formal. De esta forma, para realizar el análisis se seleccionaron sólo los(as) encuestados(as) que declararon como estado conyugal conviviente o casado.

\footnotetext{
7 "La proposición teórica que lleva a emplear esta herramienta supone que la variabilidad de la variable (estado conyugal) dependiente se puede explicar a través de las variables explicativas (edad, años de escolaridad, quintil, zona urbana o rural y condición de actividad)" (Teitelboim, 2004: 11).
} 
El modelo seleccionado para estudiar el fenómeno es la regresión logística (Agresti, 2000). Este método de regresión es conveniente cuando se cuenta con una variable dependiente de dos categorías, en este caso estar casado o convivir. Como el objetivo es determinar la probabilidad de ser conviviente, se usará la regresión logística que permite predecir probabilidades (Teitelboim, 2004).

Se optó por realizar dos modelos, uno para hombres y otro para mujeres, para así evitar el efecto de duplicidad de información debido a que la encuesta Casen se realiza para hogares, de tal forma que todos(as) los(as) encuestados(as) de la muestra tienen una relación de pareja ya sea de casados(as) o convivientes, su respuesta se verá duplicada, cuando ambos miembros de la pareja vivan en el mismo hogar, por lo tanto resta variabilidad a los datos. Por otro lado, estos dos modelos nos ayudan a reconocer diferencias entre géneros, puesto que se observa con mayor detalle el perfil de cada uno y las implicancias de ser convivientes o estar casados(as).

\subsection{Modelo para hombres}

El modelo para hombres ${ }^{8}$ es capaz de explicar un 14,1\% del fenómeno en estudio. Esto puede parecer un bajo porcentaje, pero debemos considerar que sólo incluye variables estructurales y no las subjetividades que explican la decisión de ser conviviente o casado. A través de las variables en la encuesta sólo podemos explicar la decisión de convivir o casarse a través de variables sociodemográficas.

El modelo propuesto es capaz de predecir correctamente el estado conyugal de una gran proporción de individuos (78\%), confirmando su utilidad para dar respuesta a las preguntas de investigación (ver Cuadro 5 y explicación del coeficiente de estimado para hombres en Anexo).

\subsection{Modelo para mujeres}

El modelo para mujeres ${ }^{9}$ es capaz de explicar un 15,3\% del fenómeno en estudio. Esto puede parecer un bajo porcentaje, pero al igual que en el caso anterior debemos considerar que sólo incluye variables estructurales. A través de las variables en la encuesta sólo podemos explicar la decisión de convivir o casarse a través de variables sociodemográficas.

A pesar de que el modelo presenta un bajo porcentaje de explicación, es capaz de predecir correctamente el estado conyugal de una gran proporción de individuos $(77,9 \%)$,

$8 \quad$ Una vez estimados los coeficientes del modelo se tiene que verificar si el modelo predice de manera adecuada a la variable dependiente. Verificando a través de una prueba Chi-cuadrado, podemos determinar que el modelo posee al menos una variable capaz de predecir la probabilidad de ser conviviente, por lo tanto es estadísticamente significativo con un 95\% de confianza (p-value menor a 0,05).

$9 \quad$ Una vez estimados los coeficientes del modelo, se tiene que verificar si el modelo predice de manera adecuada a la variable dependiente. Verificando a través de una prueba Chi-cuadrado, podemos determinar que el modelo posee al menos una variable capaz de predecir la probabilidad de ser conviviente, por lo tanto es estadísticamente significativo con un 95\% de confianza ( $p$-value menor a 0,05). 
confirmando su capacidad predictiva y utilidad para el estudio (ver Cuadro 6 y explicación del coeficiente de estimado para mujeres en Anexo).

Para concluir, se presenta un Cuadro Resumen (Cuadro 1) de los resultados principales de las regresiones logísticas, comparando entre hombres y mujeres. En primer lugar, las categorías edad, zona urbana y rural, condición de actividad desocupada y años de escolaridad presentan impactos levemente diferentes para hombres y mujeres sobre la variable estado conyugal. Por otro lado, estas categorías coinciden en la probabilidad de ser casados(as) o convivientes.

\section{CUADRO 1}

Principales resultados de las regresiones logísticas

\begin{tabular}{|c|c|c|}
\hline VARIABLE & MUJER & HOMBRE \\
\hline Edad & $\begin{array}{c}\text { Aumenta la probabilidad } \\
\text { de ser casada }\end{array}$ & $\begin{array}{c}\text { Aumenta la probabilidad } \\
\text { de ser casado }\end{array}$ \\
\hline Zona urbana & $\begin{array}{c}\text { Aumenta la probabilidad } \\
\text { de ser conviviente }\end{array}$ & $\begin{array}{c}\text { Aumenta la probabilidad } \\
\text { de ser conviviente }\end{array}$ \\
\hline Zona rural & $\begin{array}{c}\text { Aumenta la probabilidad } \\
\text { de ser casada }\end{array}$ & $\begin{array}{c}\text { Aumenta la probabilidad } \\
\text { de ser casado }\end{array}$ \\
\hline Condición de actividad inactiva & $\begin{array}{c}\text { Aumenta la probabilidad } \\
\text { de ser casada }\end{array}$ & $\begin{array}{c}\text { Aumenta la probabilidad } \\
\text { de ser conviviente }\end{array}$ \\
\hline $\begin{array}{l}\text { Condición de actividad } \\
\text { desocupada }\end{array}$ & $\begin{array}{c}\text { Aumenta la probabilidad } \\
\text { de ser conviviente }\end{array}$ & $\begin{array}{c}\text { Aumenta la probabilidad } \\
\text { de ser conviviente }\end{array}$ \\
\hline Años de escolaridad & $\begin{array}{c}\text { Aumenta la probabilidad } \\
\text { de ser casada }\end{array}$ & $\begin{array}{c}\text { Aumenta la probabilidad } \\
\text { de ser casado }\end{array}$ \\
\hline Quintil autónomo nacional & $\begin{array}{c}\text { Aumenta la probabilidad } \\
\text { de ser casada }\end{array}$ & No tiene efecto \\
\hline
\end{tabular}

En segundo lugar, las categorías zona urbana y condición de actividad desocupada son las únicas categorías que aumentan la probabilidad de ser conviviente. Esto se puede deber a que en centros urbanos se da con mayor frecuencia este tipo de relación, acorde a un estilo de vida más moderno. Además, las personas desocupadas son las que no tienen empleo pero han buscado trabajo en los últimos dos meses, correspondiendo a personas jóvenes (esto se reafirma al observar que a menor edad es más alta la probabilidad de ser conviviente).

En tercer lugar, las categorías edad, zona rural y años de escolaridad son las categorías que aumentan la probabilidad de ser casados(as). Esto se puede deber a que las personas mayores tienden a tener un estilo de vida más tradicional y por esta razón tienen una mayor probabilidad de estar casados(as). Además, las personas que viven en zonas rurales 
estarían menos expuestas a las nuevas tendencias de la vida moderna y las posibilidades de la convivencia como opción de pareja. Por último, las personas que tienen más años de escolaridad tienden a ser casados(as), lo que puede estar relacionado con la juventud de las personas convivientes, las cuales todavía no terminan su ciclo escolar.

En último lugar, las categorías condición de actividad inactiva y quintil autónomo nacional son las que presentan diferencias de género, puesto que las mujeres inactivas tienen mayor probabilidad de estar casadas, mientras que los hombres tienen mayor probabilidad de ser convivientes. La definición inactiva según la encuesta Casen son las personas que tienen 15 años y más y que no se encontraban ocupadas ni desocupadas, lo que significa que los hombres jóvenes que están en período escolar tienen mayor inclinación a la convivencia, y las mujeres jóvenes que están en periodo escolar tienen mayor inclinación al matrimonio. Esto se relaciona con la mayor probabilidad de estar casada si aumenta el quintil, puesto que las mujeres jóvenes casadas comparten el quintil del hogar y no individual como las mujeres que están en pareja. En cambio para los hombres el quintil no es significativo para explicar el estar casado o convivir.

Por otra parte, el hecho de que las mujeres desocupadas tiendan más a la convivencia puede estar señalando que éstas presentan un perfil que apunta a la búsqueda de inserción en el mercado laboral, a diferencia de la preponderancia de mujeres inactivas en el grupo de casadas.

\section{REFLEXIONES FINALES}

El ejercicio desarrollado en este trabajo nos lleva a reflexionar acerca de las formas en que las sociedades organizan sus modalidades de convivencia y deciden cómo constituir familias, lo cual se relaciona con los órdenes normativos, la moral, la legislación, las construcciones socioculturales acerca de lo masculino, lo femenino, la paternidad y maternidad -entre otros aspectos-. Asimismo, habla de las dinámicas de desarrollo y los modelos ideológicos que se incentivan desde distintos órdenes institucionales. En ese sentido, tanto la definición de familia como las formas en que éstas se desenvuelven en una sociedad reflejan las disputas y procesos que se juegan en el nivel simbólico y también en el desarrollo de prácticas sociales específicas de la vida cotidiana.

A partir del análisis de los datos precedentes podemos señalar, por una parte, que la sociedad chilena presenta una ampliación y diversificación de formas familiares, donde el matrimonio legalmente constituido disminuye en relación a otras modalidades de relación como las convivencias. Asimismo, podríamos hipotetizar que la mayor transversalidad de las uniones en los distintos grupos socioeconómicos es un indicador de una mayor tolerancia por parte de la sociedad a otro tipo de familias distintas a la tradicional. Ello, sumado al aumento de las uniones consensuales en grupos de distintas edades, nos permite pensar en un patrón de relación alternativo al matrimonio que se va instalando gradualmente como forma de convivencia. 
Por otra parte, se advierte que algunos factores como la vida urbana tienen un mayor nivel de explicación en estas modalidades de familia, lo que refleja que, si bien se producen cambios, éstos se presentan de manera diferenciada a lo largo del territorio. Otro elemento distintivo dice relación con el hecho de que las mujeres convivientes tienden a tener vínculo con el mercado laboral, a diferencia de las casadas; ello podría señalarnos que en la medida que aumenta la inserción de las mujeres al mercado laboral y logran una mayor estabilidad económica tienden a formar una pareja menos convencional que antaño.

A partir del análisis quedan aún muchas e importantes interrogantes respecto de la dinámica familiar, la vida privada y los vínculos entre estas dimensiones y los cambios estructurales que son importantes de abordar. ¿Qué tipos de relación se establecen entre géneros y generaciones al interior de las distintas formas familiares? ¿Cuáles son los sentidos que empujan a esta mayor diversificación en las modalidades de convivencia? ¿Qué relación existe entre los distintos tipos de inserción laboral y las formas familiares? ¿Se trata de nuevos comportamientos vinculados a una sociedad moderna, en proceso de secularización, con tendencia a la desinstitucionalización de la familia y creciente individualización? ¿Estos cambios son reflejo de una mayor tolerancia y respeto a los derechos por parte de la sociedad actual?

Pensamos para ello que el desarrollo de estudios en esta línea presentan un desafío de gran relevancia para las ciencias sociales en general y la sociología en particular, con el objeto de conocer y comprender más profundamente las dinámicas sociales actuales en nuestro país.

\section{ANEXO}

\section{CUADRO 2}

Familias casadas y convivientes 1990-2006 (porcentajes)

\begin{tabular}{|c|c|c|}
\hline AÑOS & CASADOS & CONVIVIENTES \\
\hline 1990 & 64 & 13,1 \\
\hline 2003 & 51,2 & 14,9 \\
\hline 2006 & 47,8 & 13 \\
\hline
\end{tabular}

Fuente: Casen 2006. 
CUADRO 3

Distribución de casados/as y convivientes por tramos de edad (porcentajes)

\begin{tabular}{|c|c|c|c|c|c|c|c|c|}
\hline & \multicolumn{6}{|c|}{ EDAD EN TRAMOS } & \multirow[b]{2}{*}{ TOTAL } \\
\hline & & $\begin{array}{l}18-25 \\
\text { AÑOS }\end{array}$ & $\begin{array}{l}26-35 \\
\text { AÑOS }\end{array}$ & $\begin{array}{l}36-45 \\
\text { AÑOS }\end{array}$ & $\begin{array}{l}46-55 \\
\text { AÑOS }\end{array}$ & $\begin{array}{l}56-65 \\
\text { AÑOS }\end{array}$ & $\begin{array}{c}65 \text { Y MÁS } \\
\text { AÑ̃S }\end{array}$ & \\
\hline \multirow{2}{*}{$\begin{array}{l}\text { Estado } \\
\text { conyugal } \\
\text { actual o } \\
\text { civil }\end{array}$} & Casado(a) & 2,7 & 16,2 & 27,6 & 24,4 & 16,3 & 12,7 & 100,0 \\
\hline & $\begin{array}{l}\text { Conviviente } \\
\text { o pareja }\end{array}$ & 17,4 & 29,1 & 24,1 & 16,5 & 8,2 & 4,6 & 100,0 \\
\hline \multicolumn{2}{|l|}{ Total } & 19,6 & 19,2 & 20,7 & 17,1 & 11,6 & 11,8 & 100,0 \\
\hline
\end{tabular}

Fuente: Elaboración propia en base a Casen 2006.

\section{CUADRO 4}

Distribución de casados/as y convivientes por quintiles de ingreso (porcentajes)

\begin{tabular}{|c|c|c|c|}
\hline $\begin{array}{c}\text { QUINTIL } \\
\text { AUTÓNOMO }\end{array}$ & CASADO(A) & $\begin{array}{c}\text { CONVIVIENTE O } \\
\text { PAREJA }\end{array}$ & TOTAL \\
\hline I & 18,5 & 22,9 & 19,4 \\
\hline II & 20,9 & 22,9 & 20,8 \\
\hline III & 20,4 & 20,2 & 20,8 \\
\hline IV & 20,3 & 19,3 & 20,5 \\
\hline V & 19,9 & 14,6 & 18,6 \\
\hline Total & 100,0 & 100,0 & 100,0 \\
\hline
\end{tabular}

Fuente: Elaboración propia en base a Casen 2006. 
CUADRO 5

Coeficiente de estimados para hombres

\begin{tabular}{|l|l|c|c|c|c|c|c|}
\hline \multicolumn{2}{|l|}{} & BETA & S.E. & WALD & DF & SIGNIFICACIÓN & EXP(B) \\
\hline \multirow{5}{*}{ Step 1a } & Zona (1) &,- 202 &, 004 & 2290,117 & 1 &, 000 &, 817 \\
\cline { 2 - 8 } & Quintil aut. &,- 002 &, 001 & 2,666 & 1 &, 103 &, 998 \\
\cline { 2 - 8 } & Activ. &, 504 &, 007 & 4653,381 & 1 &, 000 & 1,655 \\
\cline { 2 - 8 } & $\begin{array}{l}\text { Activ. } \\
\text { desocupado(1) }\end{array}$ &, 389 &, 005 & 5424,698 & 1 &, 000 & 1,476 \\
\cline { 2 - 8 } & $\begin{array}{l}\text { Activ. inactivo } \\
(2)\end{array}$ &,- 075 &, 000 & 31756,767 & 1 &, 000 &, 928 \\
\cline { 2 - 8 } & $\begin{array}{l}\text { Años de } \\
\text { escolaridad }\end{array}$ &,- 064 &, 000 & 233191,857 & 1 &, 000 &, 938 \\
\cline { 2 - 8 } & Edad & 2,412 &, 008 & 95381,375 & 1 &, 000 & 11,156 \\
\hline
\end{tabular}

Los coeficientes Beta de Activ. (1) y Activ. (2) son mayores a 0, por lo tanto tener una condición de actividad desocupado en comparación a ser ocupado aumenta la posibilidad de ser conviviente en 1,655 veces. De la misma manera, si se tiene como condición de actividad inactiva (en comparación de ser ocupado) la probabilidad de ser conviviente aumenta en 1,476 veces. La variable quintil no es estadísticamente significativa, lo que indica que el nivel de ingreso no es capaz de predecir si un encuestado es conviviente o casado. Por otro lado, las variables años de escolaridad, edad y zona rural y urbana son factores que disminuyen la probabilidad de ser conviviente. A mayores niveles de escolaridad y a mayor edad, la probabilidad de ser conviviente disminuye. En otras palabras, personas adultas y con más educación tienden a casarse. Para una persona cualquiera, si aumenta los años de escolaridad en un año, la probabilidad de ser conviviente disminuye en 0,928 veces. Además, una variación en la edad en un año produce una disminución en la probabilidad de ser conviviente en 0,938 veces. En el caso de zona donde habita (rural y urbana), la probabilidad de ser conviviente disminuye en 0,817 si se vive en zonas rurales (si se habita en zonas urbanas aumenta la posibilidad de ser convivientes). La variable quintil no es estadísticamente significativa, lo que indica que el nivel de ingreso no es capaz de predecir si un encuestado es conviviente o casado. 
CUADRO 6

Coeficiente de estimados para mujeres

\begin{tabular}{|l|l|c|c|c|c|c|c|}
\hline \multicolumn{2}{|l|}{} & BETA & S.E. & WALD & DF & SIGNIFICACIÓN & EXP(B) \\
\hline \multirow{5}{*}{ Step 1a } & Zona (1) &,- 235 &, 004 & 3054,264 & 1 &, 000 &, 791 \\
\cline { 2 - 8 } & Quintil aut. &,- 015 &, 001 & 161,621 & 1 &, 000 &, 985 \\
\cline { 2 - 8 } & Activ. &, 177 &, 007 & 611,743 & 1 &, 000 & 1,194 \\
\cline { 2 - 8 } & $\begin{array}{l}\text { Activ. } \\
\text { desocupada (1) }\end{array}$ &,- 295 &, 003 & 9412,270 & 1 &, 000 &, 744 \\
\cline { 2 - 8 } & Activ. inactiva (2) &,- 100 &, 000 & 50162,543 & 1 &, 000 &, 904 \\
\cline { 2 - 8 } & $\begin{array}{l}\text { Años de } \\
\text { escolaridad }\end{array}$ &,- 066 &, 000 & 254099,434 & 1 &, 000 &, 936 \\
\cline { 2 - 8 } & Edad & 2,817 &, 008 & 111379,493 & 1 &, 000 & 16,731 \\
\hline
\end{tabular}

El coeficiente Beta de Activ (1) es mayor a 0, por lo tanto tener una condición de actividad desocupada en comparación a ser ocupada aumenta la posibilidad de ser conviviente en 1,194 veces, si se mantiene el resto de las variables constantes. Por otro lado, la probabilidad de ser conviviente disminm uye si es inactiva. Por otro lado, las variables, año de escolaridad, edad, quintil autónomo y zona rural y urbana son factores que disminuyen la probabilidad de ser conviviente (aumenta la probabilidad de ser casado). Además, si aumentan los años de escolaridad, aumenta la probabilidad de ser conviviente en 0,904 veces. En cambio, si aumenta la edad, la probabilidad de ser conviviente disminuye en 0,936 veces. En el caso de zona donde habita (rural y urbana), la probabilidad de ser conviviente disminuye en 0,791 si se vive en zonas rurales (si se habita en zonas urbanas aumenta la posibilidad de ser convivientes). Por último, si aumenta el quintil la probabilidad de ser conviviente disminuye en 0,985 veces (la probabilidad de ser casado aumenta).

\section{BIBLIOGRAFÍA}

Agresti, Alan y A. Coull (2001): "Random effects modeling of multiple binary responses using the multivariate binomial logit-normal distribution", Biometrics, 57 (4).

Araujo, Kathya y Danilo Martuccelli (2012): Desafíos comunes, LOM, Santiago.

Arriagada, Irma (2001): “Familias latinoamericanas. Diagnóstico y políticas públicas en los inicios del nuevo siglo", Serie Políticas Sociales, 57, CEPAL, Santiago.

(2004): Estructuras familiares, trabajo y bienestar en América Latina, CEPAL, Santiago, disponible en http://www.familis.org/conferences/VIFamilia2005Cuba/lostrabajos/conferencia_ magistral.pdf 
Arriagada, Irma y Verónica Aranda (comps.) (2004): "Cambio de las familias en el marco de las transformaciones globales: necesidad de políticas públicas eficaces", Serie Seminarios y Conferencias, 42, CEPAL, Santiago.

Arteaga, Catalina (2007): "Prácticas familiares de reproducción en contextos de cambio", Tesis para optar al grado de Doctora en Ciencias Políticas y Sociales orientación Sociología, UNAM, México.

Beck, Ulrick y Elisabeth Beck-Gernsheim (2003): La individualización, Paidós, Madrid.

Castells, Manuel (1999): La era de la información. El poder de la identidad, Vol. II, Siglo XXI editores, España.

(2004): La era de la información, Vol. II, Alianza, España.

Cerruti, Marcela y Georgina Binstock (2009): "Familias latinoamericanas en transformación: desafíos y demandas para la acción pública", Serie Políticas Sociales, 147, CEPAL, Santiago.

De Oliveira, Orlandina y Vania Salles (1989): “Acerca del estudio de los grupos domésticos: un enfoque sociodemográfico", en Orlandina de Oliveira, Marielle Pepin Lehalleur y Vania Salles: Grupos domésticos y reproducción cotidiana, UNAM, COLMEX, Porrúa, México D.F.

García, Brígida y Olga Rojas (2002): "Cambio en la formación y disoluciones de las uniones en América Latina", Papeles de población, 32, Universidad Autónoma del Estado de México, Toluca.

González de la Rocha, Mercedes, Agustín Escobar y María de la O. Martínez Castellanos (1990): "Estrategia versus conflicto: reflexiones para el estudio del grupo doméstico en época de crisis", en Guillermo de la Peña, Juan Manuel Durán, Agustín Escobar, Javier García de Alba (comps.): Crisis, conflicto y sobrevivencia, Universidad de Guadalajara, CIESAS, México.

Harris, Cristopher Charles (1986): Familia y sociedad industrial, Península, España.

Hartmann, Heidi I. (2000): "La familia como lugar de lucha política, de género y de clase: el ejemplo del trabajo doméstico", en Marysa Navarro y Catharine R. Stimpson (comps.): Cambios sociales, económicos y culturales, FCE, México.

Jelin, Elizabeth (1984): Familia y unidad doméstica: mundo público y vida privada, CEDES, Buenos Aires. (2004): La familia en la Argentina: modernidad, crisis económica y acción política, en Bert Adams y Jan Trost (eds.): Handbook of World Families, Sage, Londres, en prensa.

Kabeer, Naila (1998), "Dictadores benevolentes, altruistas maternales y contratos patriarcales: el género y la economía doméstica", en Naila Kabeer: Realidades trastocadas. Las jerarquías de género en el pensamiento del desarrollo, Paidós, Ciudad de México.

MIDEPLAN (2006): Casen 2006, MIDEPLAN, Santiago.

Rodríguez, Jorge (2004): "Tendencias recientes de las uniones consensuales en América Latina”, Serie Seminarios y Conferencias, 42, CEPAL, Santiago.

Rosemblatt, Karin (2000): Gendered compromises: political cultures and the State in Chile, 1920-1950, University of North Carolina Press, EE.UU.

Teitelboim, Berta (2004): Factores determinantes de la pobreza en base a un modelo logístico, ICSO, UDP, Santiago.

Therborn, Göran (1999): Globalizations and Modernities. Experiences and Perspectives of Europe and Latin America, FRN, Estocolmo.

Valdés, Ximena y Teresa Valdés (eds.) (2005): Familia y vida privada ¿transformaciones, tensiones, resistencias o nuevos sentidos?, CEDEM/FLACSO, Santiago. 
Valdés, Ximena (2004): Familias en Chile: rasgos históricos y significados actuales de los cambios, CEPAL, Santiago.

(2007): La vida en común, LOM, USACH, Santiago.

Valdés, Ximena, Pamela Caro, Rosa Saavedra, Carmen Gloria Godoy, Tania Rioja, Emilie Raymond (2005): "Entre la reinvención y la tradición selectiva: familia, conyugalidad, parentalidad y sujeto en Santiago de Chile", en Ximena Valdés y Teresa Valdés (eds.): Familia y vida privada, CEDEM, FLACSO Chile, Santiago, pp. 163-214.

Wainerman, Catalina (ed.) (2003): Familia, trabajo y género: un mundo de nuevas relaciones, FCE, México.

Recibido: 05-03-2012

Aceptado: 02-08-2012 